\title{
New Random and Additional Phase Adjustment of Joint Transform Correlator
}

\author{
Man Ho Jeong* \\ Department of Laser \& Optical Information Engineering, Cheongju University, \\ 36 Naeduk-dong, Sangdang-gu, Cheongju 360-764, Korea
}

(Received January 28, 2010 : revised March 5, 2010 : accepted March 12, 2010)

\begin{abstract}
Joint transform correlator (JTC) has been the most suitable technique for real time pattern recognition. This paper proposes a new phase adjustment which adopts two steps of random phase adjustment in the spatial domain and additional phase adjustment in the Fourier domain. Simulated results are presented to show the optimum condition of the phase adjustment and the effect on the correlation peaks, the peak signal-to-noise ratio and the level of discrimination.
\end{abstract}

Keywords: Random phase adjustment, Joint transform correlator, Correlation peak

OCIS codes : (070.0070) Fourier optics and signal processing; (070.5010) Pattern recognition

\section{INTRODUCTION}

Joint transform correlator (JTC) has shown remarkable achievements and is a useful alternative to the other optical systems [1-3] for pattern recognition and target tracking applications. The typical advantages of the JTC is that it uses a type of real time optical system which quantitatively compares images by measuring correlation peaks and peak signal-to-noise ratios (PSNR). However, it has some problems on nonlinearity of the optical component such as a digital camera and on weak correlation discrimination caused by noise.

Recently, many kinds of methods for solving such problems in the JTC have been studied. Among them, the nonlinear joint transform correlator (NJTC) and the binary NJTC which can solve a problem of the nonlinear and digital components in the JTC system may be the most typical [4-7]. In spite of such efforts, a weak correlation peak and noise problems still remain. The weak correlation peak and noise problems originate from the interference fringes constructed with Fourier transformed reference and sample images in the Fourier transform plane. Some useful work has been done to solve these problems. Phase and amplitude threshold and fringe adjustment in the spatial or Fourier domains are the most typical methods [8-11].

Unlike the phase threshold technique, which uses the phase information multiplied by a simple phase function and adds a binarization step in the Fourier plane [9], we propose a new method of random and additional phase adjusted binary NJTC. The proposed new method utilizes a random phase adjustment in the first step to adjust the phase difference between the reference and input signal, and also an additional phase adjustment in the second step to minimize the total phase error. This new method shows considerably increased correlation peaks and PSNR compared with the former techniques of phase threshold multiplied by a simple phase function. Simulated results are presented to show the optimum condition of adjusting the phase function to minimize the total phase difference, and finally its effects on the correlation peaks, noise reduction and the level of discrimination.

In the following sections, the proposed new method of random and additional phase-adjusted binary NJTC system is described in detail. Section III describes simulation results and discusses the pattern recognition enhancement, and finally some comments are contained in the conclusion section.

\section{NEW RANDOM AND ADDITIONAL PHASE ADJUSTMENT}

Figure 1 shows the optical structure of the new random and additional phase-adjusted binary NJTC system which phase compensates to produce the enhanced performance compared to the conventional binary NJTC. Generally, the

\footnotetext{
*Corresponding author: manho@cju.ac.kr

Color versions of one or more of the figures in this paper are available online.
} 


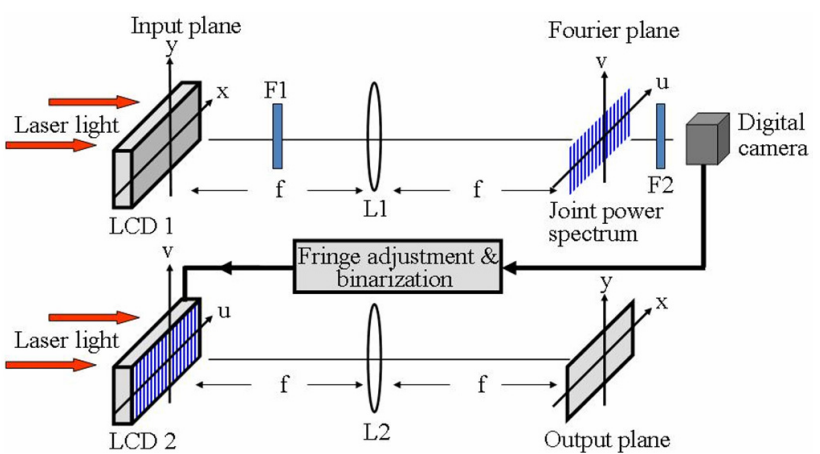

FIG. 1. Basic architecture of random and additional phase adjusted binary NJTC system.

JTC has a two-stage process. In both stages the image of a coherently illuminated input passes through a lens to take its Fourier transform. In the first stage of the JTC the basic operation is to get the information of the object Fourier transformed by Fourier transform lens L1 onto Fourier plane. Let us call the reference image $r(x, y)$ and the input image $s(x, y)$ and assume that those two inputs are separated by $2 x_{o}$. Then, the input joint images $g(x, y)$ can be expressed as

$$
g(x, y)=r\left(x+x_{0}, y\right)+s\left(x-x_{0}, y\right)
$$

The information acquired on the Fourier plane is shown as mutually interfered fringe pattern between the Fourier transformed value $R(u, v)$ of the reference image $r(x, y)$ and the Fourier transformed value $S(u, v)$ of the input image $s(x, y)$. The Fourier transformed signal of the input joint images can be expressed as

$$
G(u, v)=R(u, v) \exp \left(+j u x_{0}\right)+S(u, v) \exp \left(-j u x_{0}\right)
$$

The intensity of this interference fringe pattern is called the joint power spectrum (JPS) and can be expressed as

$$
\begin{aligned}
|G(u, v)|^{2} & =|R(u, v)|^{2}+|S(u, v)|^{2}+R(u, v) S^{*}(u, v) \exp \left(+j 2 u x_{0}\right) \\
& +R^{*}(u, v) S(u, v) \exp \left(-j 2 u x_{0}\right)
\end{aligned}
$$

Here, $*$ is the phase conjugate, $u$ and $\mathrm{v}$ are independent spatial frequency variables scaled by a factor of $2 \pi / f \lambda$, where $\lambda$ is the wavelength of input collimated light and $f$ is the focal length of the Fourier transforming lenses L1 and L2. The first and second terms in Eq. (3) are DC components which degrade the correlated signal obtained on the output plane in the second stage. DC components reconstructing a strong zero-order peak in the output plane are blocked and non-linearity parameter $k$, compensating the non-linear component problem such as a digital camera are included to obtain a well-correlated signal. Equation (4) indicates the JPS excluding the DC terms and including the non-linearity parameter $k$.

$$
\begin{aligned}
|G(u, v)|^{2} & =\left[R(u, v) S^{*}(u, v)\right]^{k} \exp \left(+j 2 u x_{0}\right) \\
& +\left[R^{*}(u, v) S(u, v)\right]^{k} \exp \left(-j 2 u x_{0}\right)
\end{aligned}
$$

Actually the JPS contains an amplitude and phase information of the reference and the input signals. Thus, Eq. (4) can be expressed as involving the amplitude and the phase of the Fourier transformed reference and input signals as

$$
\begin{aligned}
|G(u, v)|^{2}= & {\left[|R(u, v)||S(u, v)| \exp \left\{+j\left(\phi_{R}(u, v)-\phi_{S}(u, v)\right)\right\}\right]^{k} \exp \left(+j 2 u x_{0}\right) } \\
& +\left[|R(u, v)||S(u, v)| \exp \left\{-j\left(\phi_{R}(u, v)-\phi_{S}(u, v)\right)\right\}\right]^{k} \exp \left(-j 2 u x_{0}\right)
\end{aligned}
$$

Here $|G(u, v)|$ and $|S(u, v)|$ are the amplitudes of the $R(u, v)$ and $S(u, v)$, respectively, and $\phi_{R}(u, v), \phi_{S}(u, v)$, are the phases of the $R(u, v)$ and $S(u, v)$, respectively. Now, in this paper a random phase multiplication for phase adjustment was done in the spatial domain, thus Eq. (5) can be expressed as

$$
\begin{aligned}
|G(u, v)|^{2} & =\{|R(u, v)||S(u, v)|\}^{k} \exp \left\{+j\left[k\left(\phi_{R}(u, v)-\phi_{S}(u, v)+\rho_{R}(u, v)-\rho_{S}(u, v)\right)+2 u x_{0}\right]\right\} \\
& +\{|R(u, v)||S(u, v)|\}^{k} \exp \left\{-j\left[k\left(\phi_{R}(u, v)-\phi_{S}(u, v)+\rho_{R}(u, v)-\rho_{S}(u, v)\right)+2 u x_{0}\right]\right\} \\
& =2\{|R(u, v)||S(u, v)|\}^{k} \cos \left\{k\left(\phi_{R}(u, v)-\phi_{S}(u, v)+\rho_{R}(u, v)-\rho_{S}(u, v)\right)+2 u x_{0}\right\}
\end{aligned}
$$

Here, $\rho_{R}(u, v)$ and $\rho_{\mathrm{S}}(u, v)$ are the adjusted random phase of the Fourier transformed reference and input signals, respectively. In this paper, the random phase adjustment filter F1 is inserted to compensate the phase error caused by the phase difference of the reference and input images in the spatial domain. The phase error may be assumed to be caused by slight differences between the matched images and the speckle components originating from the picture of the images and the input devices, such as the LCD. These factors of the phase error have to be considered individually, but we integrated all of these factors together for developing the concepts of this paper. Now, in the case of JPS between two identical images, namely the reference and the input images are identical, the phase terms in Eq. (6) become zero. Thus, Eq. (6) can be rewritten as

$$
|G(u, v)|^{2}=2|R(u, v)|^{2 k} \cos \left(2 u x_{0}\right)
$$

Here, the adjusting random phase of the reference and the input signal are assumed to be equal. Thus in the case of two identical images, the correlation output in the output plane, namely the inverse Fourier plane, shows only two strong impulse signals separated by a distance of $4 x_{0}$. On the other hand, in the case of two different images, the correlated output shows two weak impulse signals caused by the phase difference shown in Eq. (6). 
However, in the case of two identical images it is not obvious that the correlation peaks are a maximum value in spite of the random phase adjustment as expressed in Eq. (7). Thus, the components of the phase difference expressed in Eq. (6) must be considered to calculate the correlation output. Let's consider first Eq. (4) excluding the DC terms. The output, inverse Fourier transform of Eq. (4), can be expressed as

$$
\begin{aligned}
g(x, y) & =\iint_{-\infty}^{+\infty}\left[|G(u, v)|^{2}\right] \exp (+j(u x+v y)) d u d v \\
& =(\lambda f)^{2}\left[r\left(x+2 x_{0}, y\right) \otimes s^{*}(x, y)\right]^{k} \\
& +(\lambda f)^{2}\left[r^{*}\left(x-2 x_{0}, y\right) \otimes s(x, y)\right]^{k} \\
& =(\lambda f)^{2}[|r(x, y) \| s(x, y)|]^{k} \delta\left(x+2 x_{0}, y\right) \\
& +(\lambda f)^{2}[|r(x, y) \| s(x, y)|]^{k} \delta\left(x-2 x_{0}, y\right)
\end{aligned}
$$

Here, $\otimes$ is the correlation operator. If the reference image perfectly matches the input image and there is no phase error between the reference image and the input image, the output can be expressed as

$$
\begin{aligned}
g(x, y) & =(\lambda f)^{2}|r(x, y)|^{2 k} \delta\left(x+2 x_{0}, y\right) \\
& +(\lambda f)^{2}|r(x, y)|^{2 k} \delta\left(x-2 x_{0}, y\right)
\end{aligned}
$$

Thus, if we use the phase terms as shown in Eq. (6), Equation (8) can be expressed as

$$
\begin{aligned}
g(x, y)= & \{|r(x, y)||s(x, y)|\}^{k} \iint_{-\infty}^{+\infty} \exp \left\{+j\left[k(\Delta \phi(u, v)+\Delta \rho(u, v))+2 u x_{0}\right]\right\} \\
& \times \exp (+j(u x+v y) d u d v \\
& +\{|r(x, y)||s(x, y)|\}^{k} \iint_{-\infty}^{+\infty} \exp \left\{-j\left[k(\Delta \phi(u, v)+\Delta \rho(u, v))+2 u x_{0}\right]\right\} \\
& \times \exp (+j(u x+v y) d u d v \\
= & (\lambda f)^{2}\{|r(x, y) \| s(x, y)|\}^{k} \delta\left(x+2 x_{0}, y\right) \\
& \times \iint_{-\infty}^{+\infty} \exp \{+j[k(\Delta \phi(u, v)+\Delta \rho(u, v))]\} \exp (+j(u x+v y) d u d v \\
& +(\lambda f)^{2}\{|r(x, y)||s(x, y)|\}^{k} \delta\left(x-2 x_{0}, y\right) \\
& \times \iint_{-\infty}^{+\infty} \exp \{-j[k(\Delta \phi(u, v)+\Delta \rho(u, v))]\} \exp (+j(u x+v y) d u d v
\end{aligned}
$$

Here, $\Delta \phi(u, v)=\phi_{R}(u, v)-\phi_{S}(u, v)$ and $\Delta \rho(u, v)=$ $\rho_{R}(u, v)-\rho_{S}(u, v)$. If the reference image perfectly matches the input image and there is no phase error between the reference image and the input image, the output also can be expressed as Equation (9). However, in general, there is some remaining phase error between the reference image and the input image, thus the phase terms involved in Eq. (10) would be inverse Fourier transformed, and would appear in the output plane as side-lobe noise factors around the two strong impulse signals. Therefore, in this paper another additional phase adjustment filter F2 for additional phase $\Delta \varphi_{a d d}(u, v)$ is added to reduce the remained phase error to zero and to determine its effects on the correlation peaks, noise and PSNR. Thus, the total phase in Eq. (6) can be rewritten as

$$
\text { Phase }_{\text {total }}=\Delta \phi(u, v)+\Delta \rho(u, v)+\Delta \varphi_{\text {add }}(u, v)
$$

The purpose of this paper is to minimize this total phase difference to zero to obtain the maximum correlation peaks and PSNR by reducing the phase noise involved in Eq. (10) to zero. In the next section, we present the simulated results by using the mathematical model shown in Eq. (10) and (11).

\section{COMPUTER SIMULATIONS}

To evaluate the performance of the proposed new technique of random phase adjustment in the spatial domain and additional phase adjustment in the Fourier plane, we prepared two types of $256 \times 256$ gray images of an airfield. Figure 2 shows input joint images of the reference and the input sample image. In Fig. 2, the upper part is for the matched case and the bottom part is for the mismatched case. In this paper, we introduced two widely used parameters; the correlation peaks and PSNR. Correlation peak

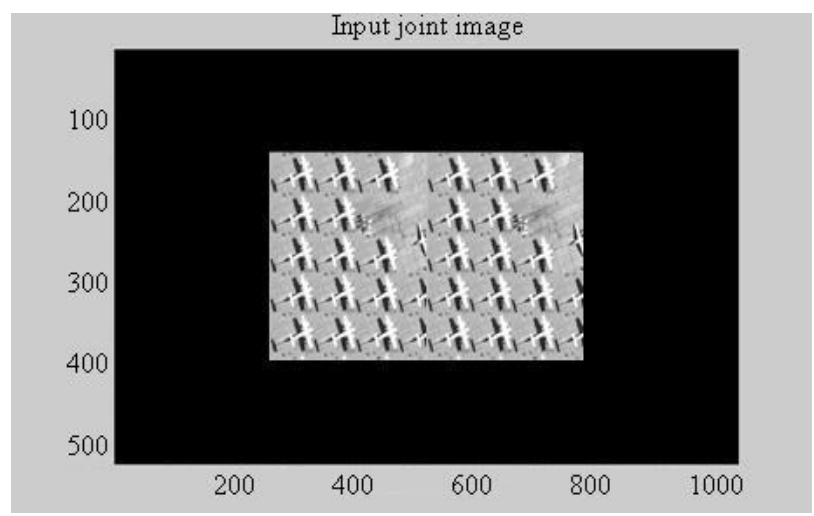

(a)

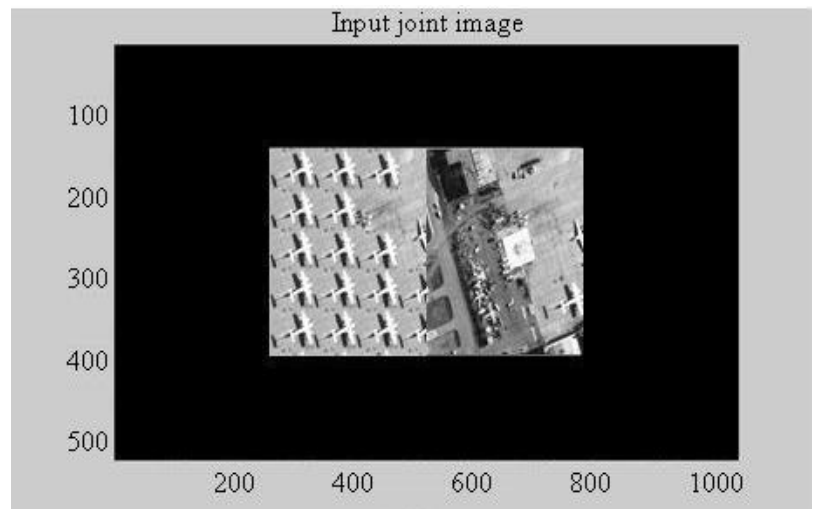

(b)

FIG. 2. 256×256 gray input joint images ; (a) matched case (b) mismatched case. 
is defined as the peak magnitude of the correlated signal and PSNR is defined as the ratio of correlation peaks to the average noise around the correlation peaks. If we let the correlation peaks equal $|p(x, y)|$, then the PSNR can be defined as.

$$
\operatorname{PSNR}=\frac{|p(x, y)|-\mu}{\sigma}
$$

Where $\mu$ represents the mean value, and $\sigma$ represents the standard deviation around the peak in the correlation output plane.

Table 1 shows the comparison of the simulation results for correlation peaks and PSNR, where $k=0.5$. The comparison was done between the conventional binary JTC and our new random phase and additional phase adjusted binary JTC. First, Table 1 indicates the correlation peaks and PSNR in the case of using the conventional binary NJTC. When the reference image is identical with the input image, namely for the matched case, the value of the correlation peaks and PSNR are $2.50 \times 10^{7}$ and $7.08 \times 10^{3}$, respectively. In contrast, when the two images are different each other, namely for the mismatched case, the value of the correlation peaks and PSNR are $1.74 \times 10^{6}$ and $6.26 \times 10^{2}$, respectively.

Second, concerning about using the proposed random phase adjustment in the spatial domain, Table 1 indicates that the whole of the correlation peaks and PSNR increased considerably when the random phase adjustment was used in place of the conventional binary NJTC. When the two images are identical each other, the value of the correlation peaks and PSNR are $8.56 \times 10^{8}$ and $8.40 \times 10^{5}$, respectively. In contrast, when the two images are different each other, the value of the correlation peaks and PSNR are
$5.99 \times 10^{8}$ and $3.34 \times 10^{5}$, respectively.

Next, concerning using the additional phase adjustment in the Fourier domain in addition to the random phase adjustment in the spatial domain, Table 1 indicates that all of the correlation peaks and PSNR increased. When the two images are identical to each other, the value of the correlation peaks and PSNR are $1.24 \times 10^{9}$ and $8.49 \times 10^{5}$, respectively. In contrast, when the two images are different fromeach other, the value of the correlation peaks and PSNR are $7.84 \times 10^{8}$ and $4.72 \times 10^{5}$, respectively. Let us pay attention to the values obtained for the correlation peaks and PSNR. From the results obtained we can find that if the conventional binary NJTC is modified with a random phase adjustment and even more with the additional phase adjustment, the correlation output shows highly improved amplitude and phase characteristics. Therefore, this result greatly improves the interference fringe pattern, and finally the correlation peaks and PSNR show more rapidly increased values. In addition, we can say finally that the noise contained in the interference fringe pattern are reduced considerably through the random and additional phase adjustment process.

Table 2 proves these facts more strongly by comparing the ratio of the correlation peaks and PSNR obtained by using both the conventional binary NJTC and the random and additional phase adjusted binary NJTC. First, in the case of the random phase adjustment, when the two images are identical, the ratio of the correlation peaks and PSNR are 35.04 and 118.64 , respectively. In contrast, when two images are different from each other, the ratio of the correlation peaks and PSNR are 344.25 and 533.55, respectively. These results reveal that PSNR shows better improvement than the correlation peaks, thus it is clear that the sidelobe noise around the correlation peaks is reduced considerably. Again, these results reveal that the discrimination ratio,

TABLE 1. Correlation peak and PSNR for the conventional binary NJTC, random phase and additional phase adjusted binary NJTC

\begin{tabular}{c|c|c|c|c|c|c}
\hline \hline \multirow{2}{*}{$\begin{array}{c}\text { Input } \\
\text { images }\end{array}$} & \multicolumn{2}{|c|}{ Conventional NJTC } & \multicolumn{2}{c|}{ Random phase adjusted NJTC } & \multicolumn{2}{c}{ Additional phase adjusted NJTC } \\
\cline { 2 - 7 } & Corr. peak & PSNR & Corr. peak & PSNR & \multicolumn{2}{c}{ Corr. peak } \\
\hline Match & $2.50 \times 10^{7}$ & $7.08 \times 10^{3}$ & $8.76 \times 10^{8}$ & $8.40 \times 10^{5}$ & $1.24 \times 10^{9}$ & $8.49 \times 10^{5}$ \\
\hline Mismatch & $1.74 \times 10^{6}$ & $6.26 \times 10^{2}$ & $5.99 \times 10^{8}$ & $3.34 \times 10^{5}$ & $7.84 \times 10^{8}$ & $4.72 \times 10^{5}$ \\
\hline
\end{tabular}

TABLE 2. Ratio of the correlation peaks and PSNR for the conventional binary NJTC, random phase and additional phase adjusted binary NJTC

\begin{tabular}{c|l|l|c|c}
\hline \hline Parameters & $\frac{(\text { Ran })_{\text {match }}}{(\text { Con })_{\text {match }}}$ & $\frac{(\text { Ran })_{\text {mismatch }}}{(\text { Con })_{\text {mismatch }}}$ & $\frac{(\text { Add })_{\text {match }}}{(\text { Con })_{\text {match }}}$ & $\frac{(\text { Add })_{\text {mismatch }}}{(\text { Con })_{\text {mismatch }}}$ \\
\hline Corr. Peaks ratio & 35.04 & 344.25 & 49.60 & 450.57 \\
\hline PSNR ratio & 118.64 & 533.55 & 119.92 & 753.99 \\
\hline
\end{tabular}

$(\text { Ran })_{\text {match }}$ : random phase adjusted, matched, (Ran $)_{\text {mismatch }}$ : mismatched

$(\text { Con })_{\text {match }}$ : conventional binary NJTC, matched, $(\text { Con })_{\text {mismatch }}$ : mismatched

$(A d d)_{\text {match }}$ : additional phase adjusted, matched, $(A d d)_{\text {mismatch }}$ : mismatched 
by which is meant the mismatching decision, was improved considerably. Second, in the case of the additional phase adjustment, when the two images are identical, the ratio of the correlation peaks and PSNR are 49.60 and 119.92, respectively. In contrast, when two images are different each other, the ratio of the correlation peaks and PSNR is 450.57 and 753.99 , respectively. These results reveal the important fact that although the total phase difference of the JPS expressed in Eq. (6) is reduced to zero as expressed in Eq. (7), there still remain some phase problems. Therefore, this kind of remaining phase problem can be removed by additional phase adjustment, and thus finally it brings an additional improve-

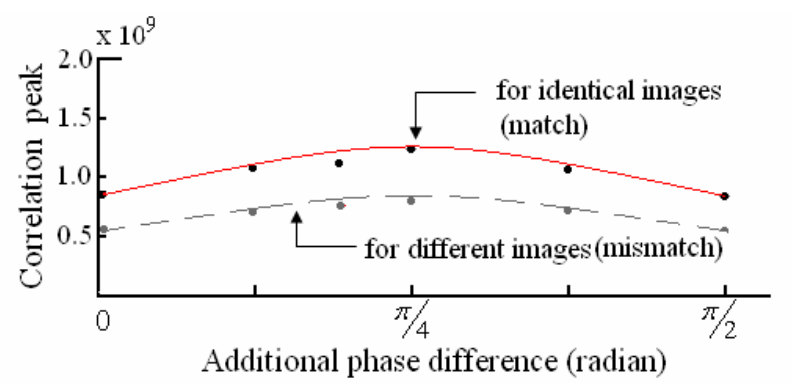

FIG. 3. Correlation peaks variation according to the additional phase adjustment.

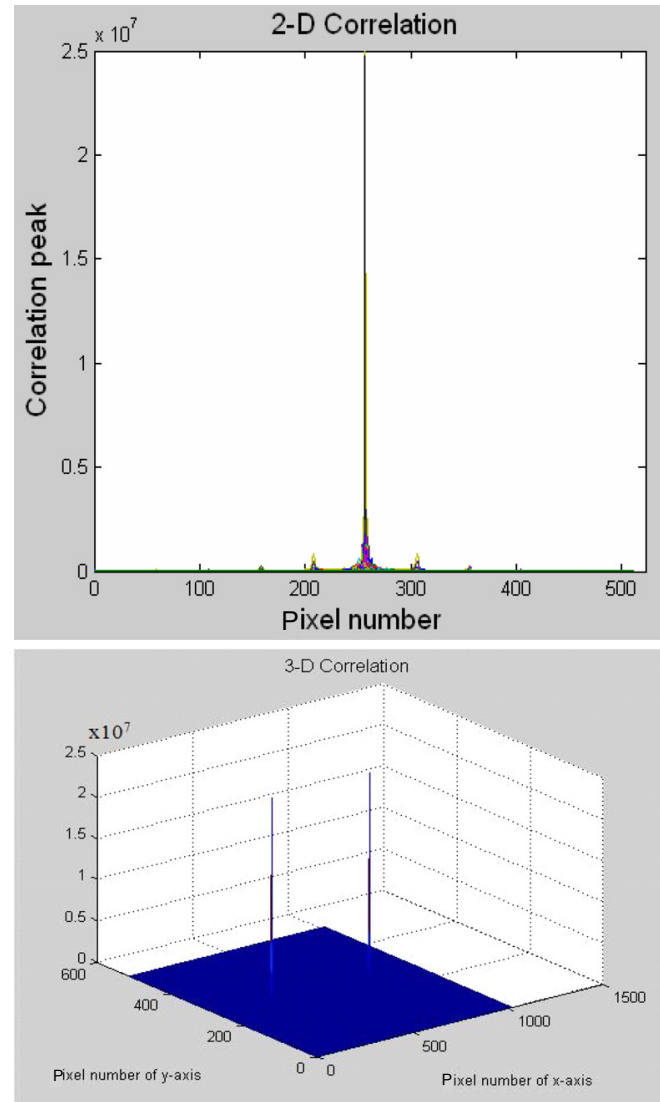

(a) ment to the correlation peaks and PSNR.

Figure 3 shows these results from a graphical point of view. In this paper, the optimum additional phase adjustment for the maximum correlation peaks is $\pi / 4$ for both matched and mismatched case. Figure 4, Fig. 5 and Fig. 6 show the two and three dimensional pictures of the correlation peaks of the matched and mismatched cases, respectively, for the conventional binary NJTC, random and additional phase adjusted binary NJTC. Two dimentional pictures in these figures represent only one of two correlation peaks separated by the distance of $4 x_{0}$.

\section{CONCLUSION}

In this paper, we presented a new technique of modification of the binary NJTC by adopting the random phase adjustment in the spatial domain and additional phase adjustment in the Fourier domain. Experimental results were presented to show that the proposed phase adjustment technique can successfully increase the correlation peaks, PSNR. In addition, final results reveal that PSNR shows more improvement than the correlation peaks, thus it is clear that the side-lobe noise around the correlation peaks is reduced considerably by using the two steps of the newly proposed phase adjustment.

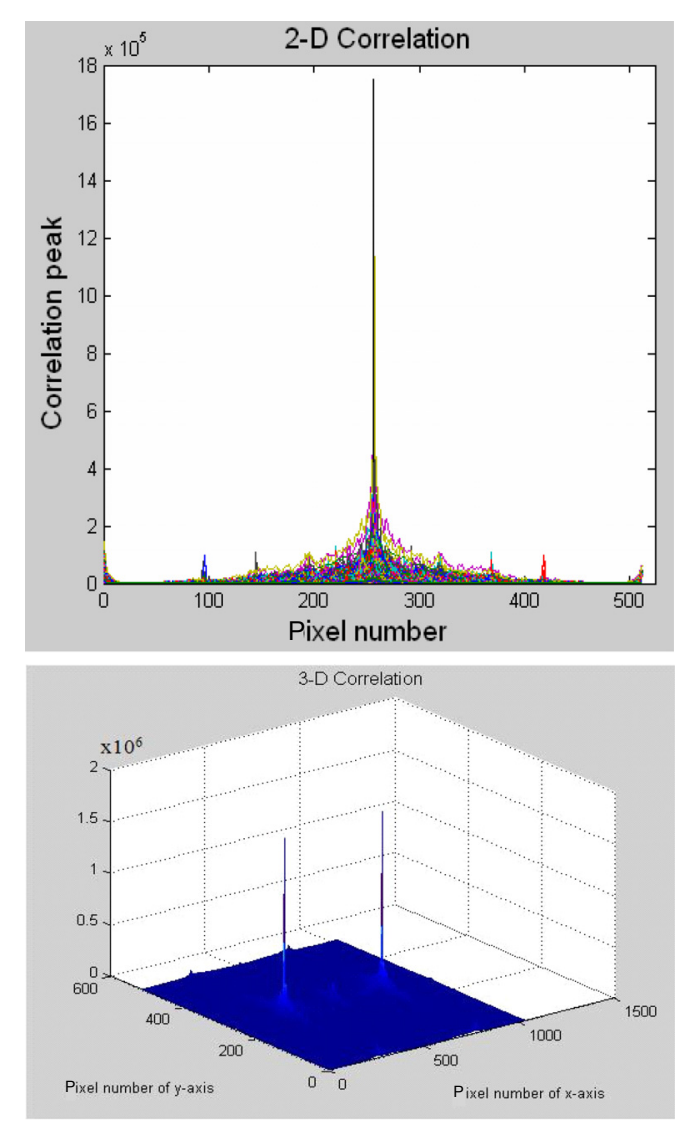

(b)

FIG. 4. 2-D and 3-D pictures of the correlation peaks for the conventional binary NJTC (a) matched (b) mismatched. 

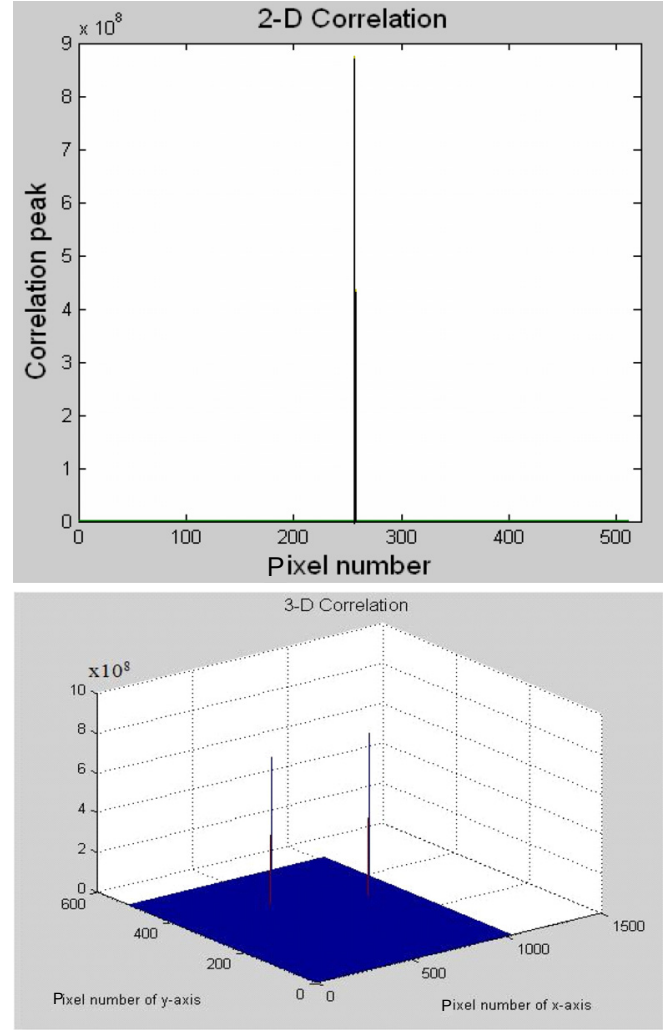

(a)
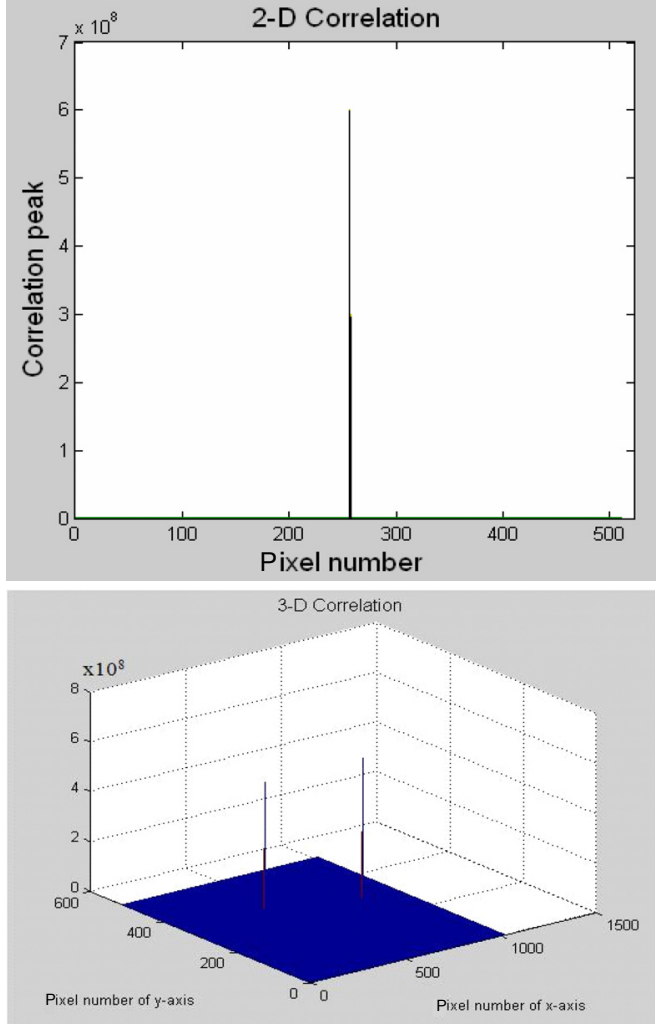

(b)

FIG. 5. 2-D and 3-D pictures of the correlation peaks for the random phase adjusted binary NJTC (a) matched (b) mismatched.
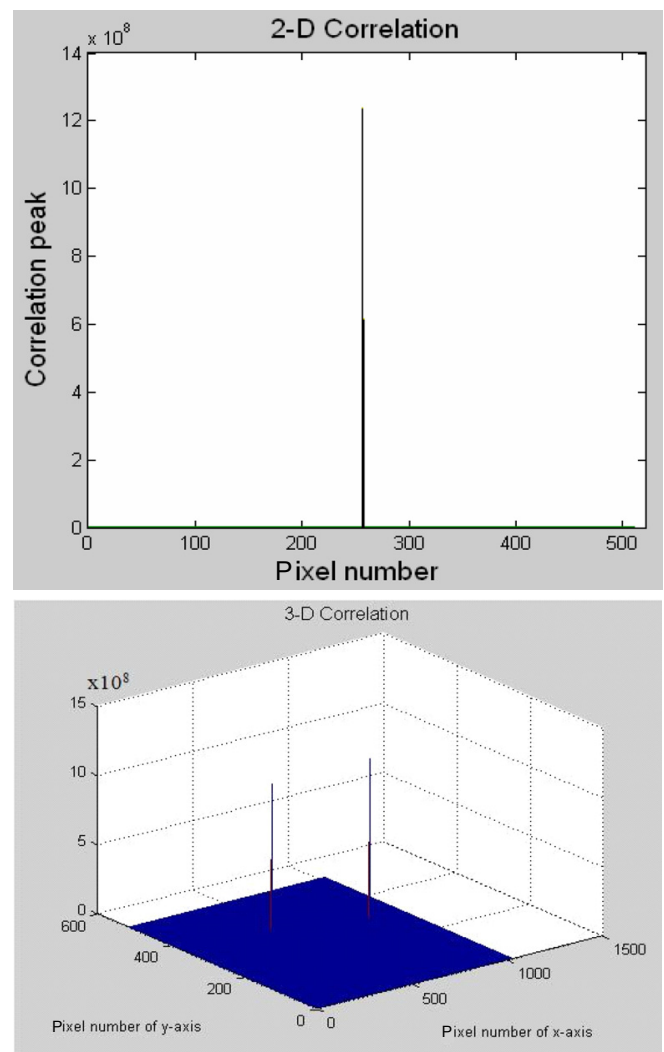

(a)
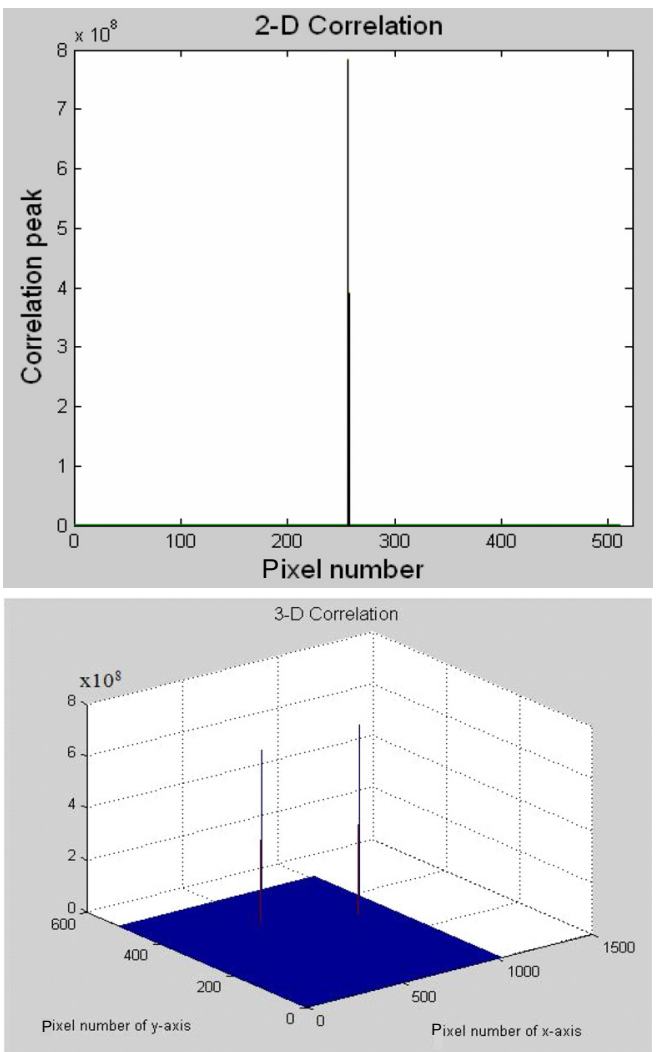

(b)

FIG. 6. 2-D and 3-D pictures of the correlation peaks for the additional phase adjusted binary NJTC (a) matched (b) mismatched. 


\section{REFERENCES}

1. C. J. Weaver and J. W. Goodman, "A technique for optically convolving two functions," Appl. Opt. 5, 1248-1249 (1966).

2. P. D. Gianino and J. L. Horner, "Phase-only matched filtering," Appl. Opt. 23, 812-816 (1984).

3. G. G. Mu, X. M. Wang, and Z. Q. Wang, "Amplitudecompensated matched filtering," Appl. Opt. 27, 3461-3463 (1988).

4. B. Javidi and J. Wang, "Binary nonlinear joint transform correlation with median and sub-set median thresholding," Appl. Opt. 30, 967-976 (1991).

5. B. Javidi, J. Li, A. H. Fazollahi, and J. Horner, "Binary nonlinear joint transform correlator performance with different thresholding methods under unknown illumination conditions," Appl. Opt. 34, 886-890 (1995).

6. M. S. Alam, A. A. S. Awwal, and M. A. Karim, "Improved correlation discrimination using joint Fourier transform optical correlator," Microwave Opt. Technol. Lett. 4, 103-106 (1991).

7. M. H. Jeong, "Analysis of fingerprint recognition characteristics based on new CGH direct comparison method and nonlinear joint transform correlator," J. Opt. Soc. Korea 13, 445-450 (2009).

8. M. S. Alam, X. W. Chen, and M. A. Karim, "Distortioninvariant fringe-adjusted joint transform correlation," Appl. Opt. 36, 7422-7427 (1997).

9. H. E. Michel and A. A. S. Awwal, "Joint Fourier transform correlation with phase thresholding in the Fourier domain," Opt. Eng. 37, 33-37 (1998).

10. M. S. Alam, J. Khan, and A. Bai, "Heteroassociative multiple-target tracking by fringe-adjusted joint transform correlation," Appl. Opt. 43, 358-365 (2004).

11. M. H. Jeong, "New iterative filter for fringe adjustment of joint transform correlator," J. Opt. Soc. Korea 14, 33-37 (2010) 